\title{
A quasi-boundary value regularization method for identifying an unknown source in the Poisson equation
}

\author{
Fan Yang*, Miao Zhang and Xiao-Xiao Li
}

"Correspondence:
yfggd114@163.com
School of Science, Lanzhou
University of Technology, Lanzhou,
Gansu 730050, People's Republic of
China
China

\begin{abstract}
In this paper, we consider the problem for identifying the unknown source in the Poisson equation in a half unbounded domain. A conditional stability result is given and a quasi-boundary value regularization method is presented to deal with this problem. For the regularization solution, the Hölder type stability estimate between the regularization solution and the exact solution is given. Numerical results are presented to illustrate the accuracy and efficiency of this method.
\end{abstract}

MSC: 35R25; 47A52; 35R30

Keywords: ill-posed problem; unknown source; conditional stability; quasi-boundary value; Poisson equation

\section{Introduction}

Inverse source problems arise in many branches of science and engineering, e.g. heat conduction, crack identification, electromagnetic theory, geophysical prospecting, and pollutant detection. For the heat source identification, there have been a large number of research results for different forms of heat source [1-9]. To the author's knowledge, there were few papers for identifying an unknown source in the Poisson equation using the regularization method. In [10], the authors identified the unknown point source with the logarithmic potential. In [11], the author identified the unknown point source using the projective method. In [12], the authors identified the unknown point source using the Green's function. In $[13,14]$, the authors identified the unknown source dependent only on one variable using the dual reciprocity method. In [15], the authors identified the unknown source dependent only on one variable using the method of fundamental solution. But by the regularization method, there are a few papers with strict theoretical analysis on identifying the unknown source.

In this paper, we consider the following inverse problem: to find a pair of functions $(u(x, y), f(x))$ which satisfy

$$
\begin{cases}-u_{x x}-u_{y y}=f(x), & -\infty<x<\infty, y>0, \\ u(x, 0)=0, & -\infty<x<\infty, \\ \left.u(x, y)\right|_{y \rightarrow \infty} \text { bounded, } & -\infty<x<\infty, \\ u(x, 1)=g(x), & -\infty<x<\infty,\end{cases}
$$

\section{Springer}

O2014 Yang et al.; licensee Springer. This is an Open Access article distributed under the terms of the Creative Commons Attribution License (http://creativecommons.org/licenses/by/2.0), which permits unrestricted use, distribution, and reproduction in any medium, provided the original work is properly cited. 
where $f(x)$ is the unknown source depending only on one spatial variable and $u(x, 1)=g(x)$ is the supplementary condition. In applications, input data $g(x)$ can only be measured, there will be measured data function $g_{\delta}(x)$ which is merely in $L^{2}(\mathbb{R})$, and it satisfies

$$
\left\|g-g_{\delta}\right\|_{L^{2}(\mathbb{R})} \leq \delta,
$$

where the constant $\delta>0$ represents a noise level of input data.

The problem (1.1) is ill-posed, i.e., the solution (if it exists) does not depend continuously on the data. One way to solve an ill-posed problem is by perturbing it into a well-posed one. Many perturbing techniques have been proposed, including a biharmonic regularization developed by Lattés and Lions in [16], a pseudo-parabolic regularization proposed by Showalter and Ting in [17], a stabilized quasi-reversibility proposed by Miller in [18], the method of quasi-reversibility proposed by Mel'nikova in [19], a hyperbolic regularization proposed by Ames and Cobb in [20], the Gajewski and Zacharias quasi-reversibility proposed by Huang and Zheng in [21], a quasi-boundary value method by Denche and Bessila in [22], and an optimal regularization proposed by Boussetila and Rebbani in [23]. It appears that Showalter in [24] was the first who used the quasi-boundary value regularization method to consider the backward heat conduction problem. In [25], the authors used the quasi-boundary-value method to consider the Cauchy problem for elliptic equations with nonhomogeneous Neumann data. In this paper, we use the quasi-boundary value regularization method to identify the unknown source for the Poisson equation.

The outline of the paper is as follows. Section 2 gives an analysis on the ill-posedness of this inverse problem and some auxiliary results. Section 3 gives a regularization solution and error estimation. Section 4 gives some examples to illustrate the accuracy and efficiency of this method. Section 5 puts an end to this paper with a brief conclusion.

\section{III-posedness of the problem and some auxiliary results}

The ill-posedness can be seen by solving the problem in the frequency domain. Let

$$
\hat{f}(\xi):=\frac{1}{\sqrt{2 \pi}} \int_{-\infty}^{\infty} e^{-i \xi x} f(x) d x
$$

be the Fourier transform of the function $f(x)$.

The problem (1.1) can be formulated in frequency space as follows:

$$
\begin{cases}\xi^{2} \hat{u}(\xi, y)-\hat{u}_{y y}(\xi, y)=\hat{f}(\xi), & \xi \in \mathbb{R}, y>0, \\ \hat{u}(\xi, 0)=0, & \xi \in \mathbb{R}, \\ \left.\hat{u}(\xi, y)\right|_{y \rightarrow \infty} \text { bounded, } & \xi \in \mathbb{R}, \\ \hat{u}(\xi, 1)=g(\xi), & \xi \in \mathbb{R} .\end{cases}
$$

The solution of the problem (1.1) is given by

$$
\hat{f}(\xi)=\frac{\xi^{2}}{1-e^{-|\xi|}} \hat{g}(\xi)
$$

So

$$
f(x)=\frac{1}{\sqrt{2 \pi}} \int_{-\infty}^{\infty} e^{i \xi x} \frac{\xi^{2}}{1-e^{-|\xi|}} \hat{g}(\xi) d \xi .
$$


The unbounded function $\frac{\xi^{2}}{1-e^{-|\xi|}}$ in (2.3) or (2.4) can be seen as an amplification factor of $\hat{g}(\xi)$ when $\xi \rightarrow \infty$. Therefore when we consider our problem in $L^{2}(\mathbb{R})$, the exact data function $\hat{g}(\xi)$ must decay rapidly as $\xi \rightarrow \infty$. But in the applications, the input data $g(x)$ can only be measured and never be exact. We assume the measured data function $g_{\delta}(x) \in$ $L^{2}(\mathbb{R})$. Thus if we try to obtain the unknown source $f(x)$, high frequency components in the error are magnified and can destroy the solution. So it is impossible to solve the problem (1.1) by using the classical method. In the following section, we will use a quasi-boundary value regularization method to deal with the ill-posed problem. Before doing that, we impose an a priori bound on the input data i.e.,

$$
\|f(\cdot)\|_{H^{p}} \leq E, \quad p>0,
$$

where $E>0$ is a constant, $\|\cdot\|_{H^{p}}$ denotes the norm in Sobolev space $H^{p}(\mathbb{R})$ defined by

$$
\|f(\cdot)\|_{H^{p}}:=\left(\int_{-\infty}^{\infty}|\hat{f}(\xi)|^{2}\left(1+\xi^{2}\right)^{p} d \xi\right)^{\frac{1}{2}}
$$

Now we give some lemmas which are very useful for our main conclusion.

Lemma 2.1 If $x>1$, we have the following inequality:

$$
\frac{1}{1-e^{-x}} \leq \frac{e}{e-1}
$$

Lemma 2.2 For $0<\beta<1$, the following inequality hold:

$$
\sup _{\xi \in \mathbb{R}}\left|\frac{\beta^{2} \xi^{2}}{\beta^{2} \xi^{2}+1-e^{-|\xi|}}\left(1+\xi^{2}\right)^{-\frac{p}{2}}\right| \leq 2 \max \left\{\beta^{p}, \beta^{2}\right\} .
$$

Proof Let

$$
A(\xi):=\frac{\beta^{2} \xi^{2}}{\beta^{2} \xi^{2}+1-e^{-|\xi|}}\left(1+\xi^{2}\right)^{-\frac{p}{2}} .
$$

The proof of (2.8) is separated into three cases.

Case 1. $|\xi| \geq \xi_{0}=\frac{1}{\beta}$; we get

$$
A(\xi) \leq\left(1+\xi^{2}\right)^{-\frac{p}{2}} \leq|\xi|^{-p} \leq \xi_{0}^{-p}=\beta^{p} .
$$

Case $2.1<|\xi|<\xi_{0}$; we obtain

$$
A(\xi) \leq \frac{\beta^{2} \xi^{2-p}}{1-e^{-|\xi|}} \leq 2 \beta^{2} \xi^{2-p} .
$$

If $0<p \leq 2$, the above inequality becomes

$$
A(\xi) \leq 2 \beta^{2} \xi^{2-p} \leq 2 \beta^{2} \xi_{0}^{2-p}=2 \beta^{p} .
$$


If $p>2$, we get

$$
A(\xi) \leq 2 \beta^{2} \xi^{2-p} \leq 2 \beta^{2}
$$

Case 3. $|\xi| \leq 1$; we get

$$
A(\xi) \leq \frac{\beta^{2} \xi^{2}}{1-e^{-|\xi|}} \leq \beta^{2} \frac{e}{e-1} \leq 2 \beta^{2}
$$

Combining (2.10) with (2.12), (2.13) and (2.14), the inequality (2.8) holds.

\section{The conditional stability result}

Since the problem (1.1) is linear, stability estimates can be derived by estimating the size of solutions to the corresponding homogeneous problem. We establish the stability estimate for the problem (1.1).

Theorem 3.1 Suppose that $f(x)$ is the solution of the problem (1.1) with the exact data $g(x)$, and suppose that (2.5) holds; then the following estimate holds:

$$
\|f(\cdot)\| \leq \frac{e}{e-1}\|g(\cdot)\|+E^{\frac{2}{p+2}}\left(\frac{e}{e-1}\right)^{\frac{p}{p+2}}\|g(\cdot)\|^{\frac{p}{p+2}} .
$$

Proof According to (1.2), and using the Parseval formula, we have

$$
\|f(\cdot)\|^{2}=\|\hat{f}(\cdot)\|^{2}=\int_{|\xi| \leq 1}\left|\frac{\xi^{2}}{1-e^{-|\xi|}}\right|^{2}|\hat{g}(\xi)|^{2} d \xi+\int_{|\xi| \geq 1}|\hat{f}(\xi)|^{2} d \xi=: A_{1}+A_{2} .
$$

According to (2.7), when $|\xi| \leq 1$, we obtain

$$
\left|\frac{\xi^{2}}{1-e^{-|\xi|}}\right| \leq \frac{e}{e-1}
$$

Hence

$$
A_{1} \leq\left(\frac{e}{e-1}\right)^{2}\|g(\cdot)\|^{2}
$$

Now for $A_{2}$, using the Hölder inequality, we have

$$
\begin{aligned}
A_{2} & =\int_{|\xi| \geq 1}|\hat{f}(\xi)|^{2} d \xi=\int_{|\xi| \geq 1}\left[\left(1+|\xi|^{2}\right)^{p}|\hat{f}(\xi)|^{2}\right]^{\frac{2}{p+2}}\left[\left(1+|\xi|^{2}\right)^{-2}|\hat{f}(\xi)|^{2}\right]^{\frac{p}{p+2}} d \xi \\
& \leq\left(\int_{|\xi| \geq 1}\left(1+|\xi|^{2}\right)^{p}|\hat{f}(\xi)|^{2} d \xi\right)^{\frac{2}{p+2}}\left(\int_{|\xi| \geq 1}\left(1+|\xi|^{2}\right)^{-2}|\hat{f}(\xi)|^{2} d \xi\right)^{\frac{p}{p+2}} \\
& \leq\|f(\cdot)\|_{H^{p}}^{\frac{4}{p+2}}\left(\int_{|\xi| \geq 1}\left(1+|\xi|^{2}\right)^{-2}\left|\frac{\xi^{2}}{1-e^{-|\xi|}} \hat{g}(\xi)\right|^{2} d \xi\right)^{\frac{p}{p+2}} \\
& \leq\|f(\cdot)\|_{H^{p}}^{\frac{4}{p+2}}\left(\sup _{|\xi| \geq 1} \frac{1}{\left(1+|\xi|^{2}\right)^{2}}\left|\frac{\xi^{2}}{1-e^{-|\xi|}}\right|^{2}\right)^{\frac{p}{p+2}}\left(\int_{-\infty}^{+\infty}|\hat{g}(\xi)|^{2} d \xi\right)^{\frac{p}{p+2}}
\end{aligned}
$$




$$
\begin{aligned}
& =\|f(\cdot)\|_{H^{p}}^{\frac{4}{p+2}}\left(\sup _{|\xi| \geq 1} \frac{|\xi|^{2}}{\left(1+|\xi|^{2}\right)^{2}}\left|\frac{1}{1-e^{-|\xi|}}\right|^{2}\right)^{\frac{p}{p+2}}\|g(\cdot)\|^{\frac{2 p}{p+2}} \\
& \leq\|f(\cdot)\|_{H^{p}}^{\frac{4}{p+2}}\left(\frac{e}{e-1}\right)^{\frac{2 p}{p+2}}\|g(\cdot)\|^{\frac{2 p}{p+2}} \cdot
\end{aligned}
$$

So we obtain

$$
\begin{aligned}
\|f(\cdot)\| & \leq \sqrt{A_{1}+A_{2}} \leq \sqrt{A_{1}}+\sqrt{A_{2}} \\
& \leq \frac{e}{e-1}\|g(\cdot)\|+E^{\frac{2}{p+2}}\left(\frac{e}{e-1}\right)^{\frac{p}{p+2}}\|g(\cdot)\|^{\frac{p}{p+2}} .
\end{aligned}
$$

Remark 3.1 Suppose the functions $f_{1}(x)$ and $f_{2}(x)$ are the solutions of the problem (1.1) with the exact data $g_{1}(x)$ and $g_{2}(x)$, respectively, then we have the estimate

$$
\left\|f_{1}(\cdot)-f_{2}(\cdot)\right\| \leq \frac{e}{e-1}\left\|g_{1}(\cdot)-g_{2}(\cdot)\right\|+E^{\frac{2}{p+2}}\left(\frac{e}{e-1}\right)^{\frac{p}{p+2}}\left\|g_{1}(\cdot)-g_{2}(\cdot)\right\|^{\frac{p}{p+2}} .
$$

From (3.3), it is obvious that $\left\|f_{1}(\cdot)-f_{2}(\cdot)\right\| \rightarrow 0$ when $\left\|g_{1}(\cdot)-g_{2}(\cdot)\right\| \rightarrow 0$. However, this conditional stability result cannot ensure the stability of numerical computation with noisy data. We must use the regularization method to deal with this ill-posed problem.

\section{The quasi-boundary value regularization method and the error estimate}

To obtain a stable approximate solution of the problem (1.1), we make a modification of the boundary value of the (1.1) as follows:

$$
u(x, 1)+\beta^{2} f(x)=g_{\delta}(x) .
$$

We can obtain the regularization solution of the problem (1.1) by solving the following problem:

$$
\begin{cases}-u_{x x}-u_{y y}=f(x), & -\infty<x<\infty, y>0, \\ u(x, 0)=0, & -\infty<x<\infty, \\ \left.u(x, y)\right|_{y \rightarrow \infty} \text { bounded, } & -\infty<x<\infty \\ u(x, 1)+\beta^{2} f(x)=g_{\delta}(x), & -\infty<x<\infty\end{cases}
$$

where the parameter $\beta$ is regarded as a regularization parameter. The problem (4.2) can be formulated in frequency space as follows:

$$
\begin{cases}\xi^{2} \hat{u}(\xi, y)-\hat{u}_{y y}(\xi, y)=\hat{f}(\xi), & \xi \in \mathbb{R}, y>0, \\ \hat{u}(\xi, 0)=0, & \xi \in \mathbb{R}, \\ \left.\hat{u}(\xi, y)\right|_{y \rightarrow \infty} \text { bounded, } & \xi \in \mathbb{R}, \\ \hat{u}(\xi, 1)+\beta^{2} \hat{f}(\xi)=\hat{g}_{\delta}(\xi), & \xi \in \mathbb{R} .\end{cases}
$$

The solution of the problem (4.3) is given by

$$
\hat{f}_{\beta, \delta}(\xi)=\frac{\xi^{2}}{\beta^{2} \xi^{2}+1-e^{-|\xi|}} \hat{g}_{\delta}(\xi),
$$


i.e.,

$$
f_{\beta, \delta}(x)=\frac{1}{\sqrt{2 \pi}} \int_{-\infty}^{\infty} e^{i \xi x} \frac{\xi^{2}}{\beta^{2} \xi^{2}+1-e^{-|\xi|}} \hat{g}_{\delta}(\xi) d \xi
$$

Note that for small $\beta, \frac{\xi^{2}}{\beta^{2} \xi^{2}+1-e^{-|\xi|}}$ is close to $\frac{\xi^{2}}{1-e^{-|\xi|}}$. On the other hand, if $|\xi|$ becomes large, $\frac{\xi^{2}}{\beta^{2} \xi^{2}+1-e^{-|\xi|}}$ is bounded. So $f_{\beta, \delta}(x)$ is considered as an approximation of $f(x)$.

Now we will give an error estimate between the regularization solution and the exact solution by the following theorem.

Theorem 4.1 Let $f(x)$ given by (2.4) be the exact solution of (1.1) and let $f_{\beta, \delta}(x)$ given by (4.5) be its regularization solution. Let assumptions (1.2) and an a priori condition (2.5) hold. If we select

$$
\beta=\left(\frac{\delta}{E}\right)^{\frac{1}{p+2}},
$$

then we have the following error estimate:

$$
\left\|f(\cdot)-f_{\beta, \delta}(\cdot)\right\| \leq \delta^{\frac{p}{p+2}} E^{\frac{2}{p+2}}\left(1+2 \max \left\{1,\left(\frac{\delta}{E}\right)^{\frac{2-p}{p+2}}\right\}\right) .
$$

Proof Using the Parseval formula, the triangle inequality, (2.8), and (4.6), we obtain

$$
\begin{aligned}
& \left\|f(\cdot)-f_{\beta, \delta}(\cdot)\right\|=\left\|\hat{f}(\cdot)-\hat{f}_{\beta, \delta}(\cdot)\right\| \\
& =\left\|\frac{\xi^{2}}{1-e^{-|\xi|}} \hat{g}(\xi)-\frac{\xi^{2}}{\beta^{2} \xi^{2}+1-e^{-|\xi|}} \hat{g}_{\delta}(\xi)\right\| \\
& \leq\left\|\frac{\xi^{2}}{1-e^{-|\xi|}} \hat{g}(\xi)-\frac{\xi^{2}}{\beta^{2} \xi^{2}+1-e^{-|\xi|}} \hat{g}(\xi)\right\| \\
& +\left\|\frac{\xi^{2}}{\beta^{2} \xi^{2}+1-e^{-|\xi|}} \hat{g}(\xi)-\frac{\xi^{2}}{\beta^{2} \xi^{2}+1-e^{-|\xi|}} \hat{g}_{\delta}(\xi)\right\| \\
& =\left\|\frac{\xi^{2}}{1-e^{-|\xi|}} \hat{g}(\xi)\left(1+\xi^{2}\right)^{\frac{p}{2}}\left(1+\xi^{2}\right)^{-\frac{p}{2}}\left(1-\frac{1-e^{-|\xi|}}{\beta^{2} \xi^{2}+1-e^{-|\xi|}}\right)\right\| \\
& +\left\|\frac{\xi^{2}}{\beta^{2} \xi^{2}+1-e^{-|\xi|}}\left(\hat{g}(\xi)-\hat{g}_{\delta}(\xi)\right)\right\| \\
& \leq\left\|\hat{f}(\xi)\left(1+\xi^{2}\right)^{\frac{p}{2}}\left(\frac{\beta^{2} \xi^{2}}{\beta^{2} \xi^{2}+1-e^{-|\xi|}}\left(1+\xi^{2}\right)^{-\frac{p}{2}}\right)\right\| \\
& +\sup _{\xi \in \mathbb{R}}\left|\frac{\xi^{2}}{\beta^{2} \xi^{2}+1-e^{-|\xi|}}\right|\left\|\hat{g}(\xi)-\hat{g}_{\delta}(\xi)\right\| \\
& \leq \sup _{\xi \in \mathbb{R}}\left|\frac{\beta^{2} \xi^{2}}{\beta^{2} \xi^{2}+1-e^{-|\xi|}}\left(1+\xi^{2}\right)^{-\frac{p}{2}}\right|\left\|\hat{f}(\xi)\left(1+\xi^{2}\right)^{\frac{p}{2}}\right\| \\
& +\sup _{\xi \in \mathbb{R}}\left|\frac{\xi^{2}}{\beta^{2} \xi^{2}+1-e^{-|\xi|}}\right| \delta \\
& \leq 2 \max \left\{\beta^{p}, \beta^{2}\right\} E+\frac{1}{\beta^{2}} \delta
\end{aligned}
$$




$$
\begin{aligned}
& =2 \max \left\{\left(\frac{\delta}{E}\right)^{\frac{p}{p+2}},\left(\frac{\delta}{E}\right)^{\frac{2}{p+2}}\right\} E+\left(\frac{\delta}{E}\right)^{\frac{-2}{p+2}} \delta \\
& =\delta^{\frac{p}{p+2}} E^{\frac{2}{p+2}}\left(1+2 \max \left\{1,\left(\frac{\delta}{E}\right)^{\frac{2-p}{p+2}}\right\}\right) .
\end{aligned}
$$

\section{Several numerical examples}

In this section, we present three numerical examples to illustrate the usefulness of the proposed method. The numerical examples were constructed in the following way: First we selected the source function $f(x)$, and we obtained the exact data function $g(x)$ by solving the direct problem. Then we added a normally distributed perturbation to each data function and obtained vectors $g_{\delta}(x)$. Finally we obtained the regularization solution $f_{\beta, \delta}(x)$ by solving the inverse problem.

In the following, we first give an example which has the exact expression of the solutions $(u(x, y), f(x))$.

Example 1 It is easy to see that the function

$$
u(x, y)=\left(1-e^{-y}\right) \sin x
$$

and the function

$$
f(x)=\sin x
$$

are satisfied with the problem (1.1) with the exact data

$$
g(x)=\left(1-e^{-1}\right) \sin x .
$$

Suppose that the sequence $\left\{g_{k}\right\}_{k=0}^{n}$ represents samples from the function $g(x)$ on an equidistant grid, then we add a random uniformly perturbation to each data, which forms the vector $g_{\delta}$, i.e.,

$$
g_{\delta}=g+\varepsilon \operatorname{randn}(\operatorname{size}(g))
$$

where

$$
g=\left(g\left(x_{1}\right), \ldots, g\left(x_{n}\right)\right)^{T}, \quad x_{i}=(i-1) \Delta x, \Delta x=\frac{1}{n-1}, i=1,2, \ldots, n
$$

The function 'randn $(\cdot)$ ' generates arrays of random numbers whose elements are normally distributed with mean 0 , variance $\sigma^{2}=1$. ' $\operatorname{randn}(\operatorname{size}(g))$ ' returns an array of random entries that is the same size as $g$. The total noise level $\delta$ can be measured in the sense of root mean square error (RMSE) according to

$$
\delta=\left\|g_{\delta}-g\right\|_{l^{2}}=\left(\frac{1}{n} \sum_{i=1}^{n}\left(g_{i}-g_{i, \delta}\right)^{2}\right)^{\frac{1}{2}}
$$


Figure 1 Comparison between the exact solution (- -$)$ and its computed approximations with various noise levels of $\varepsilon=0.01(-*-)$, $\varepsilon=0.001(-\triangleright-), \varepsilon=0.0001(-\circ-)$ for Example 1: (a) $p=2$, (b) $p=3$, (c) $p=4$.

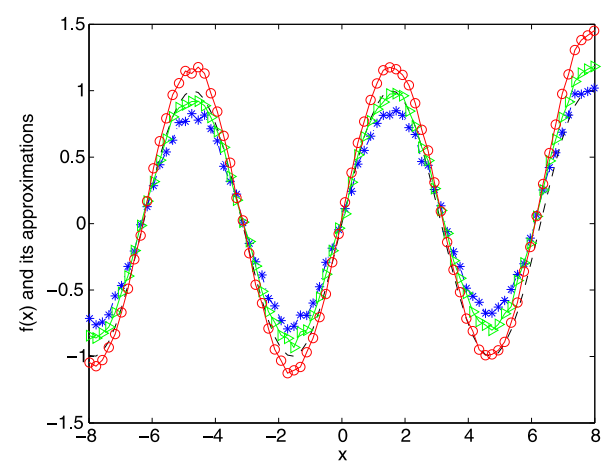

(a)

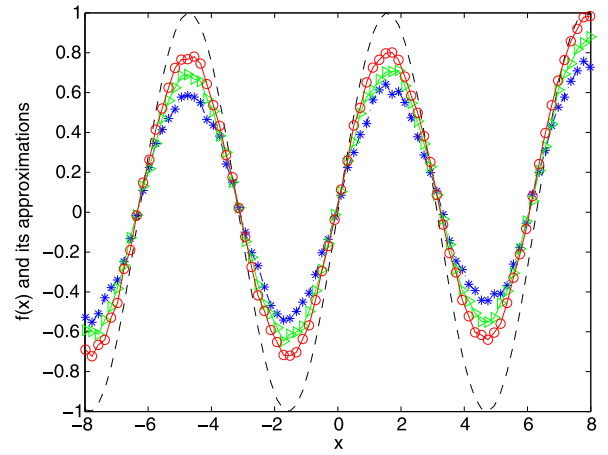

(b)

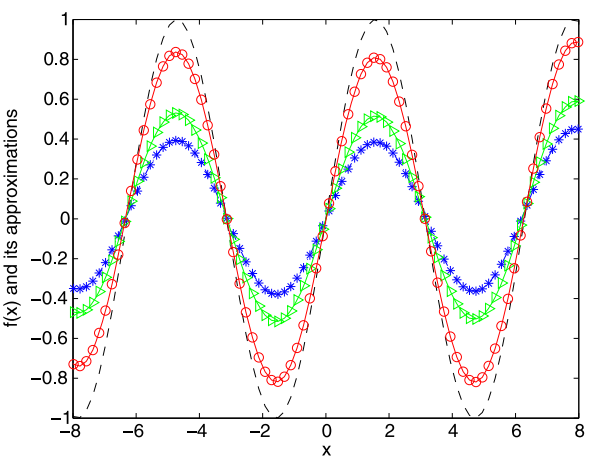

(c)

Moreover, we need to make the vector $g_{\delta}$ periodical [26] and then we take the discrete Fourier transform for the vector $g_{\delta}$. The approximation of the regularization solution are computed by using the fast Fourier transform algorithm [26] and the range of the variable $x$ in the numerical experiment is $[-8,8]$.

Example 2 Consider a piecewise smooth source:

$$
f(x)= \begin{cases}0, & -8 \leq x \leq-4 \\ x+4, & -4<x \leq 0 \\ -x+4, & 0<x \leq 4 \\ 0, & 4<x \leq 8\end{cases}
$$


Figure 2 Comparison between the exact solution and its computed approximations with various levels of noise for Example 2: (a) $p=2$, (b) $p=3$, (c) $p=4$.

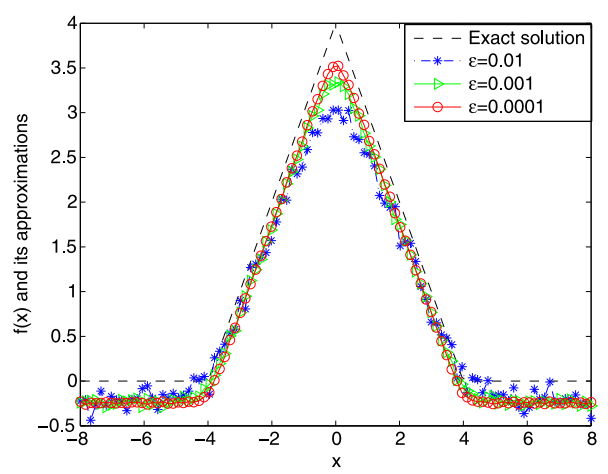

(a)

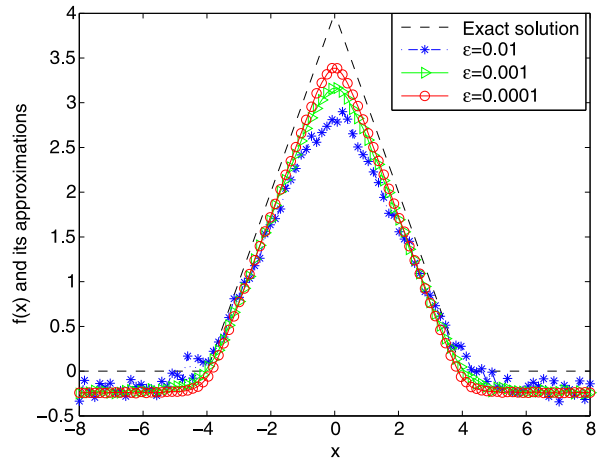

(b)

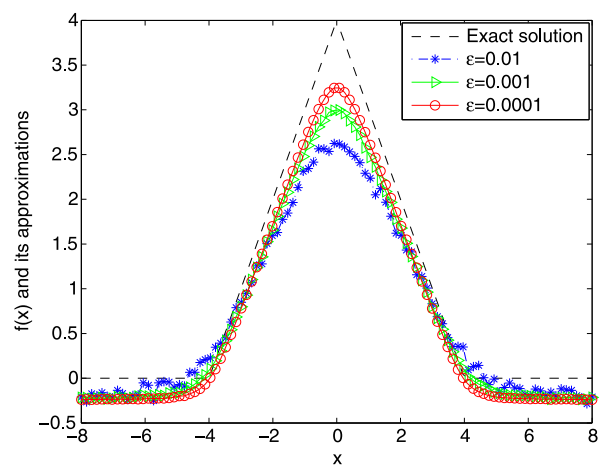

(c)

Example 3 Consider the following discontinuous case:

$$
f(x)= \begin{cases}-1, & -8 \leq x \leq-4, \\ 1, & -4<x \leq 0, \\ -1, & 0<x \leq 4, \\ 1, & 4<x \leq 8 .\end{cases}
$$

From Figures 1-3, we can see that the smaller the $\varepsilon$, the better the computed approximation $f_{\beta, \delta}(x)$. As $p$ increases, the worse the computed approximation. This means the numerical results are not so good for stronger 'smoothness' assumptions on the exact solution $f(x)$ which is consistent with the Tikhonov regularization method in [27]. 
Figure 3 Comparison between the exact solution and its computed approximations with various levels of noise for Example 3: (a) $p=2$, (b) $p=3$, (c) $p=4$.

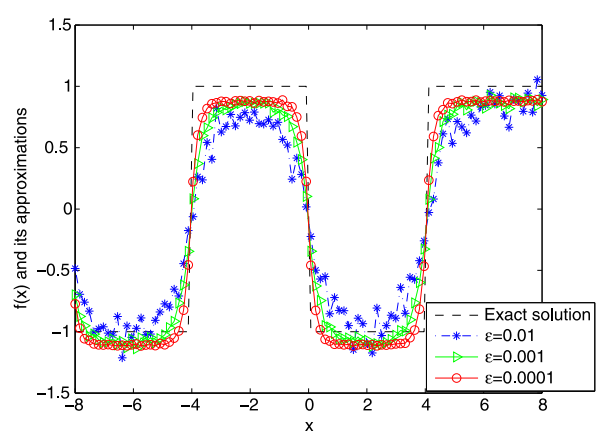

(a)

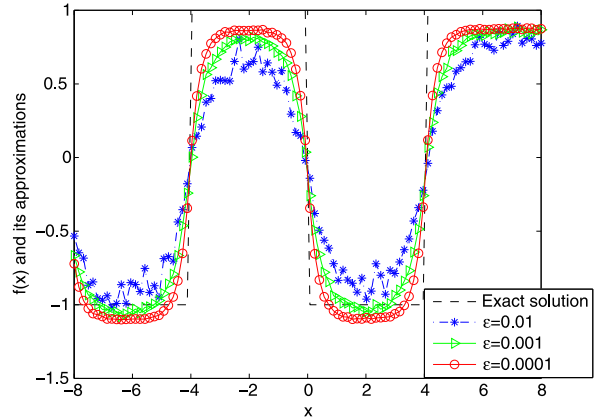

(b)

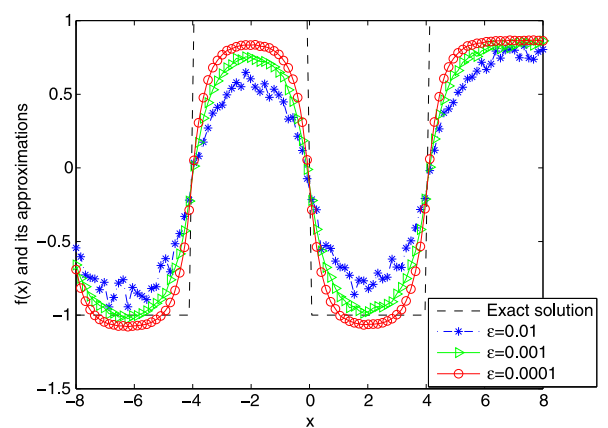

(c)

In Examples 2 and 3, since the direct problem with the source $f(x)$ does not have an analytical solution, the data $g(x)$ is obtained by solving the direct problem. From Figures 2 and 3, we can see that the numerical solutions are less ideal than that of Example 1. It is not difficult to see that the well-known Gibbs phenomenon and the recovered data near the non-smooth and discontinuities points are not accurate. Taking into consideration of the ill-posedness of the problem, the results presented in Figures 2 and 3 are reasonable.

\section{Conclusions}

In this paper, we identify an unknown source term depending only on one variable in twodimensional Poisson equation. This problem is ill-posed, i.e., the solution (if it exists) does not depend on the input data. We obtained the stability estimate using the conditional stability. Moreover, using the quasi-boundary value regularization method, we obtain the regularization solution and the Hölder type error estimate between the exact solution and the regularization solution. According to [28], this Hölder type error estimate is order optimal. 


\section{Competing interests}

The authors declare that they have no competing interests.

\section{Authors' contributions}

All authors contributed equally to the writing of this paper. All authors read and approved the final manuscript.

\section{Acknowledgements}

The project is supported by the National Natural Science Foundation of China (No. 11171136, No. 11261032), the Distinguished Young Scholars Fund of Lanzhou University of Technology (Q201015) and the Basic Scientific Research Business Expenses of Gansu Province College.

\section{Received: 15 October 2013 Accepted: 7 March 2014 Published: 20 Mar 2014}

\section{References}

1. Ahmadabadi, MN, Arab, M, Malek Ghaini, FM: The method of fundamental solutions for the inverse space-dependent heat source problem. Eng. Anal. Bound. Elem. 33, 1231-1235 (2009)

2. Cannon, JR, Duchateau, P: Structural identification of an unknown source term in a heat equation. Inverse Probl. 14, 535-551 (1998)

3. Yan, L, Fu, CL, Yang, FL: The method of fundamental solutions for the inverse heat source problem. Eng. Anal. Bound. Elem. 32, 216-222 (2008)

4. Dou, FF, Fu, CL, Yang, FL: Optimal error bound and Fourier regularization for identifying an unknown source in the heat equation. J. Comput. Appl. Math. 230, 728-737 (2009)

5. Wei, T, Zhang, ZQ: Reconstruction of a time-dependent source term in a time-fractional diffusion equation. Eng. Anal. Bound. Elem. 37, 23-31 (2013)

6. Li, GS: Data compatibility and conditional stability for an inverse source problem in the heat equation. Appl. Math. Comput. 173, 566-581 (2006)

7. Ma, YJ, Fu, CL, Zhang, YX: Identification of an unknown source depending on both time and space variables by a variational method. Appl. Math. Model. 36, 5080-5090 (2012)

8. Yang, L, Deng, ZC, Yu, JN, Luo, GW: Optimization method for the inverse problem of reconstructing the source term in a parabolic equation. Math. Comput. Simul. 80, 314-326 (2009)

9. Yang, F, Fu, CL: Two regularization methods to identify time-dependent heat source through an internal measurement of temperature. Math. Comput. Model. 53, 793-804 (2011)

10. Ohe, T, Ohnaka, K: A precise estimation method for locations in an inverse logarithmic potential problem for point mass models. Appl. Math. Model. 18, 446-452 (1994)

11. Nara, T, Ando, S: A projective method for an inverse source problem of the Poisson equation. Inverse Probl. 19 355-369 (2003)

12. Farcas, A, Elliott, L, Ingham, DB, Lesnic, D, Mera, NS: A dual reciprocity boundary element method for the regularized numerical solution of the inverse source problem associated to the Poisson equation. Inverse Probl. Sci. Eng. 11(2), 123-139 (2003)

13. Kagawa, Y, Sun, Y, Matsumoto, O: Inverse solution of Poisson equation using DRM boundary element models identification of space charge distribution. Inverse Probl. Sci. Eng. 1(2), 247-265 (1995)

14. Sun, Y, Kagawa, Y: Identification of electric charge distribution using dual reciprocity boundary element models. IEEE Trans. Magn. 33(2), 1970-1973 (1997)

15. Jin, BT, Marin, L: The method of fundamental solutions for inverse source problems associated with the steady-state heat conduction. Int. J. Numer. Methods Eng. 69, 1570-1589 (2007)

16. Lattés, R, Lions, JL: The Method of Quasireversibility: Applications to Partial Differential Equations. Elsevier, New York (1969)

17. Showalter, RE, Ting, TW: Pseudo-parabolic partial differential equations. SIAM J. Math. Anal. 1(1), 1-26 (1970)

18. Miller, K: Stabilized quasireversibility and other nearly best possible methods for non-well posed problems. In: Symposium on Non-Well-Posed Problems and Logarithmic Convexity. Lecture Notes in Math., vol. 316, pp. 161-176. Springer, Berlin (1973)

19. Mel'nikova, IV: Regularization of ill-posed differential problems. Sib. Math. J. 33(2), 289-298 (1992)

20. Ames, KA, Cobb, SS: Continuous dependence on modeling for related Cauchy problems of a class of evolution equations. J. Math. Anal. Appl. 215(1), 15-31 (1997)

21. Huang, Y, Zheng, Q: Regularization for a class of ill-posed Cauchy problems. Proc. Am. Math. Soc. 133(10), 3005-3012 (2005)

22. Denche, M, Bessila, K: A modified quasi-boundary value method for ill-posed problems. J. Math. Anal. Appl. 301(2)، 419-426 (2005)

23. Boussetila, N, Rebbani, F: Optimal regularization method for ill-posed Cauchy problems. Electron. J. Differ. Equ. 2006 147 (2006)

24. Showalter, RE: Cauchy problem for hyperparabolic partial differential equations. In: Trends in the Theory and Practice of Non-Linear Analysis, pp. 421-425. Elsevier, Amsterdam (1983)

25. Feng, XL, Eldén, L, Fu, CL: A quasi-boundary-value method for the Cauchy problem for elliptic equations with nonhomogeneous Neumann data. J. Inverse III-Posed Probl. 18(6), 617-645 (2010)

26. Eldén, L, Berntsson, F, Regiǹska, T: Wavelet and Fourier methods for solving the sideways heat equation. SIAM J. Sci. Comput. 21, 2187-2205 (2000)

27. Kirsch, A: An Introduction to the Mathematical Theory of Inverse Problems. Springer, New York (1996)

28. Engl, HW, Hanke, M, Neubauer, A: Regularization of Inverse Problem. Kluwer Academic, Boston (1996)

10.1186/1029-242X-2014-117

Cite this article as: Yang et al.: A quasi-boundary value regularization method for identifying an unknown source in

the Poisson equation. Journal of Inequalities and Applications 2014, 2014:117 